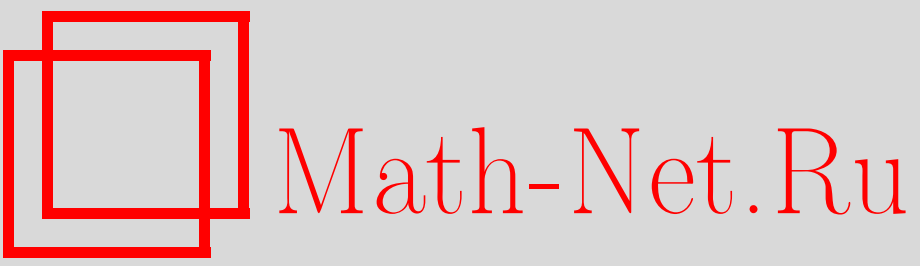

Н. П. Долбилин, М. А. Штанько, М. И. Штогрин, Неизгибаемость зоноэдров, УМН, 1996, том 51, выпуск 2, 157-158

DOI: https://doi.org/10.4213/rm954

Использование Общероссийского математического портала Math-Net.Ru подразумевает, что вы прочитали и согласны с пользовательским соглашением

http://www.mathnet.ru/rus/agreement

Параметры загрузки:

IP : 54.147 .182 .235

26 апреля 2023 г., 15:13:16 


\title{
НЕИЗГИБАЕМОСТЬ ЗОНОЭДРОВ
}

\author{
Н. П. ДолБИлин, М. А. ШтАнькО, М. И. ШтОГРИн
}

В настоящей заметке обобщается теорема о неизгибаемости квадрильяжей двумерной сферы $S^{2}$ в $\mathbf{E}^{3}$, изложенная в [3]. А именно, мы покажем неизгибаемость очень широкого класса невыпуклых многогранников, введенных Е. С. Федоровым еще в 1885 году [2], так называемых зоноэдров, вложенных в 3 -мерное евклидово пространство.

С основными понятиями, результатами и проблемами теории изгибания поверхностей можно познакомиться в сборнике [1].

Здесь под зоноәдром мы будем понимать абстрактно заданную полиэдральную сферу такую, что: а) каждая грань является евклидовым выпуклым многоугольником с четным числом сторон; б) множество сторон каждого многоугольника разбивается на пары сторон, параллельных между собой.

Для таких многогранников естественно вводится понятие пояса или зоны [2, с. 256]. Квадрильяж сферы $S^{2}$ есть важный частный случай зоноэдров Федорова и понятие полоски, служившей полезным инструментом для изучения свойств квадрильяжа, совпадает с понятием пояса для произвольных зоноэдров.

Зоноэдр $Q$, вложенный в $\mathbf{E}^{3}$ (с плоскими гранями), называется неизгибаемылм, если существует такое $\varepsilon>0$, что любой изометричный ему (с теми же плоскими гранями) зоноэдр $Q^{\prime} \subset \mathbf{E}^{3}$ при условии $\left\|Q-Q^{\prime}\right\|<\varepsilon$ конгруэнтен $Q$.

ТеОремА. Зоноэдр, вложенный в $\mathbf{E}^{3}$, является неизгибаемым.

Рассмотрим графб отмеченных ребер Г, который был введен в [3]. Допустим, что вложенный зоноэдр допускает непрерывное изгибание $\varphi$, в процессе которого каждая грань остается плоской. Тогда в процессе изгибания $\varphi$ хотя бы один двугранный угол, т.е. угол между плоскостями двух смежных граней, должен изменяться. Назовем всякое ребро изменяющегося при изгибании $\varphi$ двугранного угла отмеченным. Рассмотрим изгибание $\varphi$ и будем предполагать его настолько малым, что вложенный зоноэдр при изгибании $\varphi$ остается по-прежнему вложенным. Обозначим граф̆, состоящий из всех отмеченных ребер, через $Г$. Ребра граффа $\Gamma$ - это отмеченные ребра зоноэдра, а вершины граффа - это вершины отмеченных ребер и только они.

Перечислим некоторые свойства графа отмеченных ребер. Заметим, что множество ребер графа Г непусто тогда и только тогда, когда зоноэдр $Q$ допускает изгибание $\varphi$.

Г1. Граф Г разбивает поверхность зоноэдра на несколько компонент связности. Каждое отмеченное ребро разделяет две различные компоненты, т.е. является границей двух граней зоноэдра, принадлежащих разным компонентам.

Г2. В каждой вериине графа Г должно встречаться не менее двух ребер. Это немедленно следует из Г1.

ГЗ. Если в вериине сходятся только два отмеченных ребра, то они лежат на прямой.

Г4. В вершине графа Г сходятся либо 2 ребра, либо не меньше 4.

Доказательство этих свойств графа Г см. в [3].

Перейдем от графа отмеченных ребер к графу $\Gamma_{\varphi}$, который будем называть графом изгибания $\varphi$. Вершинами графа изгибания $\Gamma_{\varphi}$ объявляются лишь те вершины графа $\Gamma$, в которых сходятся не менее 4 отмеченных ребер. Рассмотрим произвольное отмеченное ребро $e$ из исходного графа $Г$ и выделим те отмеченные ребра, которые можно соединить с $e$ реберным путем, проходящим через вершины графа $Г$ степени 2 . В силу свойства $Г 3$, а также ограниченности зоноэдра, объединение таких ребер образует прямолинейный отрезок, который мы будем считать ребром "нового" графа $\Gamma_{\varphi}$. Оба конца такого ребра являются вершинами валентности не ниже 4 , т.е. вершинами нового графа. Приведем необходимые нам свойства графа изгибания.

$\Gamma_{\varphi} 1$. Все ребра графа $\Gamma_{\varphi}$ суть прямолинейнье отрезки, состоящие из нескольких отмеченных ребер.

$\Gamma_{\varphi}$ 2. Никакие два ребра графа $\Gamma_{\varphi}$ не имеют два общих конца.

Работа выполнена при частичной поддержке Российского фонда фундаментальных исследований (грант № 96-01-00166) и Немецкого научно-исследовательского общества (DFG). 
Это вытекает из того, что зоноэдр $Q$ вложен в $\mathbf{E}^{3}$. Таким образом, в графе изгибания нет двуреберных циклов.

$\Gamma_{\varphi} 3$. В каждой вершине графа $\Gamma_{\varphi}$ сходятся не менее 4 ребер.

$\Gamma_{\varphi} 4$. Граф Гч разбивает поверхность зоноэдра на области, среди которых имеется по крайней мере восемь треугольников (под треугольником понимается область, граница которой состоит из трех и только трех ребер графа $\left.\Gamma_{\varphi}\right)$.

В самом деле, обозначим число компонент графа $\Gamma_{\varphi}$ через $k$, число его вершин - через $v$, число ребер - через $е$ и, наконец, число областей, на которые сфера разбивается ребрами графа $\Gamma_{\varphi}$, - через $f$. Заметим, что область может быть и не диском, а ограничивающий ее реберный контур, состоящий из прямолинейных отрезков, может быть не связным. Обозначим через $f_{n}$ число областей с $n$ ребрами. Тогда можно написать следующие соотношения:

$$
\sum n f_{n} \geqslant 4 v, \quad \sum n f_{n}=2 e, \quad \sum f_{n}=f .
$$

Из этих соотношений и обобщенной формулы Эйлера $(v-e+f=k+1)$ получаем:

$$
\sum(4-n) f_{n} \geqslant 4(v-e+f) \geqslant 4 \cdot 2 .
$$

Отсюда выводим, что $f_{3} \geqslant 8+f_{5}+2 f_{6}+\cdots+(n-4) f_{n}+\cdots$. Таким образом, в графе $\Gamma_{\varphi}$ имеется, по крайней мере, восемь треугольников.

ЗАмечАниЕ. Из приведенных рассуждений фактически следует, что каждая связная компонента графа $\Gamma_{\varphi}$ содержит не менее восьми треугольников.

ДокАЗАТЕЛЬСтво тЕОРЕмЫ. Предположим противное: вложенный в $\mathbf{E}^{3}$ зоноэдр $Q$ допускает непрерывное изгибание $\varphi$. Тогда должен существовать граф изгибания $\Gamma \varphi$. Обозначим через $\Delta$ одну из треугольных областей зоноэдра $Q$ (см. $\left.\Gamma_{\varphi} 4\right)$, и пусть $a$ - какое-нибудь ребро, которое лежит на стороне треугольника $\Delta$. Пояс (в смысле Федорова), построенный по ребру $a$, содержит две грани, скажем, $F_{1}$ и $F_{2}$, примыкающие к ребру $a$, причем одна из них, пусть $F_{1}$, лежит вне треугольника $\Delta$, в то время как другая грань, $F_{2}$, лежит внутри $\Delta$. Двигаясь вдоль этого пояса в направлении от $F_{1}$ к $F_{2}$, мы встретим первую после $F_{2}$ грань $F_{k}$, которая уже не принадлежит треугольнику $\Delta$. Тогда предыдущая грань $F_{k-1}$ в поясе лежит внутри $\Delta$. Так как грани $F_{k-1}$ и $F_{k}$ имеют лишь одно общее ребро $b$ и принадлежат одному поясу, то ребро $b$ принадлежит контуру $\Delta$ и проходит параллельно ребру $a$. Поэтому отмеченные ребра $a$ и $b$ не могут лежать на разных сторонах треугольника $\Delta$, т.е. обязаны лежать на одной стороне треугольника. Однако ребра $a$ и $b$ не могут лежать и на одной стороне. Полученное противоречие показывает, что никакой граф $\Gamma_{\varphi}$ со свойствами $\Gamma_{\varphi} 1-\Gamma_{\varphi} 4$ не может быть вложен в реберный остов зоноэдра $Q$. Таким образом, предположение об изгибании привело к противоречию, что доказывает теорему.

ЗАмЕчАниЕ 1. Хотя в теореме говорится о неизгибаемости вложенных зоноэдров, в действительности же, как видно из доказательства, неизгибаемыми являются и погруженные зоноэдры, а также такие зоноэдры, которые отображены в $\mathbf{E}^{3}$ линейно на каждой грани и чьи одномерные (реберные) остовы погруженъ в $\mathbf{E}^{3}$.

ЗАмечАние 2. Рассмотрим разбиение $\mathbf{E}^{3}$ на федоровские параллелоэдры, т.е. на 3 -мерные выпуклые многогранники, заполняющие пространство при помощи параллельных переносов. Как известно, существует 5 аффинных типов таких параллелоэдров. Пусть $Q$ - полиэдральная сфе$\mathrm{pa}$, составленная из граней 2-мерного остова разбиения пространства на параллелоэдры. Тогда полиэдральная сфера $Q$ является зоноэдром и по доказанной теореме неизгибаема.

\section{СПИСОК ЛИТЕРАТУРЫ}

[1] Исследования по метрической теории поверхностей. Сборник статей. Серия Математика. Новое в зарубежной науке. Т. 18. М.: Мир, 1980. [2] Федоров Е. С. Начала учения о фигурах. Л.: Изд-во АН СССР, 1953. [3] Долбилин Н. П., Штанько М. А., Штогрин М. И. Неизгибаемость квадрильяжа сферы // ДАН. 1996 (в печати). 